\title{
Influence of the naturally occurring human CASP1 variant L265S on subcellular distribution and pyroptosis
}

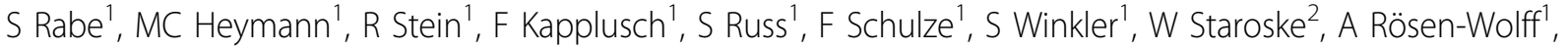 \\ SR Hofmann ${ }^{1 *}$
}

From 8th International Congress of Familial Mediterranean Fever and Systemic Autoinflammatory Diseases Dresden, Germany. 30 September - 3 October 2015

\section{Introduction}

Patients with unexplained recurrent febrile episodes and CASP1 variants suffer from systemic sterile inflammation despite altered enzymatic activity of procaspase-1 and reduced IL- $1 \beta$ release. Most recent findings from our group indicate that the proinflammatory effects of CASP1 variants with reduced or abrogated enzymatic activity could be due to receptor interacting protein kinase 2 (RIP2) mediated increase of NF-kB activation. These findings are additionally supported by a trend to elevated IL- 6 and TNF- $\alpha$ expression in patients with CASP1 variants.

\section{Objectives}

The objective of this project is the identification of possible subcellular mechanisms how the CASP1-L265S variant influences proinflammatory cell death (pyroptosis) and IL-1 $\beta$ secretion.

\section{Methods}

We established an in vitro model of a virally transduced human monocytic cell line (THP-1 with shRNA knockdown of endogenous procaspase-1), expressing either wild type or enzymatically inactive (L265S) procaspase-1 fusion-reporter proteins and characterized them after NLRP3-inflammasome stimulation. Using confocal microscopy and in vivo live cell imaging we analyzed the subcellular distribution of fluorescently labeled procaspase-1 wildtype and variant as well as the interaction with ASC.

\section{Results}

First results revealed a disturbed nuclear localization of the CASP1-L265S variant compared to procaspase-1 wildtype. Furthermore, CASP1-L265S variant revealed a strongly decreased pyroptosome formation and a less intense interaction with ASC (apoptosis-associated speck-like protein containing a CARD) after NLRP3stimulation. Variant procaspase-1 L265S and ASC formed smaller pyroptosomes than wildtype procaspase-1and ASC.

\section{Conclusion}

Those findings suggest a model, in which variant procaspase-1 L265S impairs nuclear localization, pyroptosome formation and ASC-interaction, leading all together to reduced IL-1 $\beta$ production and secretion.

\section{Authors' details \\ ${ }^{1}$ Technische Universität Dresden, Department of Pediatrics, Medizinische Fakultät Carl Gustav Carus, Dresden, Germany. ${ }^{2}$ Technische Universität Dresden, Biotechnology Center, Dresden, Germany.}

Published: 28 September 2015

doi:10.1186/1546-0096-13-S1-061

Cite this article as: Rabe et al:: Influence of the naturally occurring human CASP1 variant L265S on subcellular distribution and pyroptosis. Pediatric Rheumatology 2015 13(Suppl 1):O61. 